\title{
Submicron Structure in Biocompatible Ferrofluids
}

\author{
G. Matei $^{a}$, A. Airinei $^{b}$ And D.-E. CreangA ${ }^{a}$ \\ ${ }^{a}$ Univ. Al. I. Cuza, Fac. of Physics, Iasi, Romania \\ ${ }^{b}$ P. Poni Macromolecular Chemistry Institute, Iasi, Romania
}

This paper presents a comparative study between the microstructural features of two water-ferrofluids intended for biological applications. In both cases the ferrophase was prepared by auto-catalytic reaction between ferrous and ferric salts and resulted in magnetite and some maghemite precipitates. The difference is given by the stabilizer molecule: tetramethylammonium hydroxide and, respectively, citric acid. Transmission electron microscopy and atomic force microscopy were utilized for ferrophase size investigation. The evidence of short chains and large aggregates was obtained mainly by atomic force microscopy analysis (the tip diameter being equal to $5 \mathrm{~nm}$ ). Their role in the ferrofluid rheological properties was studied by carrying out comparative measurements of viscosity and surface tension. Complementary investigation of the ferrofluid composition was done by means of IR absorption spectra. The suitability of the prepared ferrofluids for biological goals was proved by their convenient ferrophase diameter as well as by their stability in time.

PACS numbers: 68.37.Lp, 68.37.Ps, 78.30.-j

\section{Introduction}

Submicron magnetic particles suspended in suitable carrier fluids are consistent with ferrofluid colloids already known for their various technical and medical uses. However, the preparation protocol, though based on the apparently simple co-precipitation method, remains a rather complex procedure from the viewpoint of the stability control of the final product. Especially, the stability of water ferrofluids intended for medical use is a current issue of frontier research. The adequate choice of the ferrophase coating technology can ensure the compensation of the attractive forces between ferrophase particles avoiding the formation of large aggregates that precipitate and destabilize the colloidal material. Afterwards the analysis of the ferrophase size and ferrofluid rheological features was done for two water-ferrofluids stabilized with different coating molecules: the tetramethylammonium hydroxide and the citric acid. 


\section{Material and methods}

\subsection{Ferrofluid}

The ferrophase was prepared from pure heptahydrate of $\mathrm{FeSO}_{4}$ and hexahydrate of $\mathrm{Fe}_{2} \mathrm{Cl}_{3}$ salts following auto-catalysis reaction [1] in alkali reaction medium (25\% ammonium hydroxide in excess); ferrophase coated with citric acid (CA) and, respectively, tetramethylammonium hydroxide (TMA) was dispersed in water (prepared according to $[2,3]$ ) at $80^{\circ} \mathrm{C}$ with non-magnetic stirring.

\subsection{Microstructural investigation}

Atomic force microscopy (AFM) device used for this investigation works in the tapping mode with commercial standard silicon nitride cantilever (NSC21) having a force constant of $17.5 \mathrm{~N} \mathrm{~m}^{-1}, 210 \mathrm{kHz}$ resonance frequency, and tips with radii between 10 and $20 \mathrm{~nm}$. The AFM images cover a range of areas, from $50 \times 50$ to $3 \times 3 \mu \mathrm{m}^{2}$. The ferrofluid dispersion (both diluted and non-diluted samples) was deposited on mica substrate.

Electron microscopy was applied using Tesla transmission electron microscopy (TEM) device $(40000 \times)$ having a resolution of $1.0 \mathrm{~nm}$, the ferrofluid being diluted $10^{-4}$ in water and deposited on collodion sheet.

The IR investigation was carried out using a Perkin Elmer 580 device on the dispersion in $\mathrm{KBr}$ of the ferrofluid sample previously thermally treated at $100^{\circ} \mathrm{C}$ up to constant weight.

Viscosity measurements were carried out by capillary method (Ostwald viscosimeter). The surface tension measurements were performed by applying the stalagmometric method. Repeated measurements on the same ferrofluid sample gave statistical assurance. All necessary reactives were reactive grade.

\section{Results and discussion}

Preliminary investigation on the ferrophase diameter has been accomplished by means of AFM scanning. In Fig. 1 some isolated particles as well as particle aggregates are visible. Their diameter is ranging between 10 and $1000 \AA$ while their height is up to $200 \AA$.

In detailed picture the accuracy of the physical diameter measurement is suggested (Fig. 1b).

The distribution histograms could be entirely analysed after TEM picture analysis (Fig. 2). As in the case of AFM images no qualitative differences between TEM images of the two ferrofluids have been noticed. We have revealed bi-modal histograms, the maximum frequency of the small particle sub-population (average diameter of $9 \mathrm{~nm}$ ) being twice higher than that of the large particle sub-population (average diameter of about $100 \mathrm{~nm}$ ). As already stated, the optimal size of ferrophase particles is governed by the conditions of energy balance (magnetic dipole-magnetic dipole interaction, inhomogeneous magnetic field energy, gravitational field energy, and thermal movement energy) [4] the size of ferrophase 

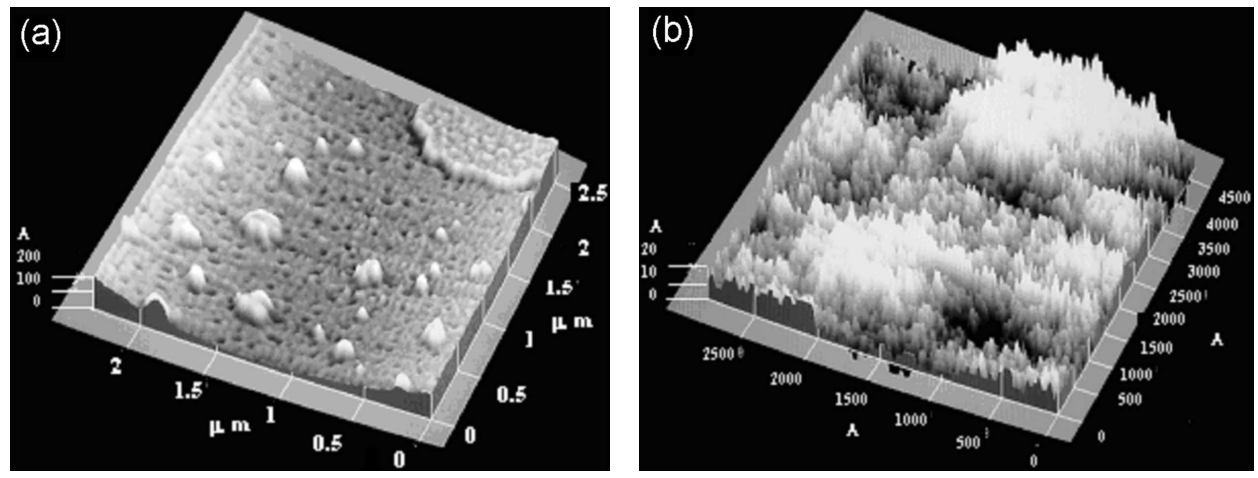

Fig. 1. (a) Particles and particle aggregates in the ferrophase of citric acid ferrofluid deposited on mica support; (b) the detailed picture of AFM 3D scan.

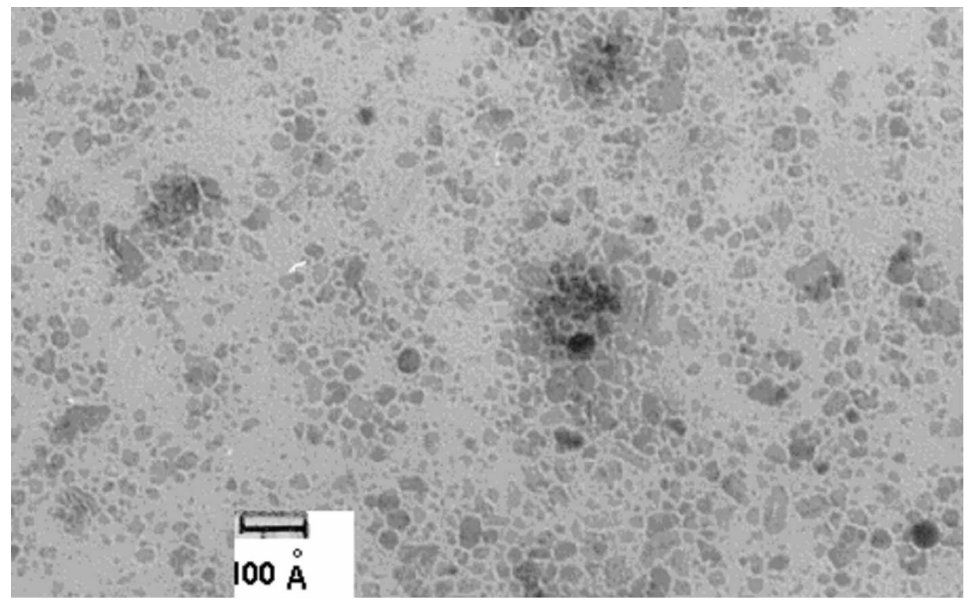

Fig. 2. TEM picture of ferrophase particles and particle agglomerates.

particles being recommended to be lower than $8 \mathrm{~nm}$ or even $7.8 \mathrm{~nm}$. Such ferrofluids behave as stable magnetic colloids suitable for technical and biomedical applications. In the case of biomedical applications the biocompatibility requirements impose water as carrier fluid and consequently certain long chain surfactant molecules are able to stabilize the colloidal dispersion against the gravitational or magnetic sedimentation. We have used the citric acid in parallel with the most concentrated hydroxide of tetramethylammonium to coat ferrophase particles.

What is more, the chemical biocompatibility is related to certain ferrophase structure, namely magnetite - that we have prepared by auto-catalytic reaction which does not assure satisfactory control upon the ferrophase particle diameter. Therefore, the submicron colloidal particles that we have obtained are characterized not by uniform diameter size but by log normal distribution. Since the magnetic attraction forces are difficult to balance, the presence of particle ag- 
gregates and short chains is practically unavoidable - the minimization of their percentage being the only realistic aim. The AFM scanning has revealed the topology of such aggregates and very short chains which are not overlapped but only interconnected into the same plane: the height of the aggregates being smaller than their diameter and comparable with the diameter of isolated particles. TEM images, though unable to provide 3D view, can emphasize better the particles that are smaller than the AFM tip diameter.

Further analysis was carried out by means of IR spectra. The IR spectrum (4000-400 $\mathrm{cm}^{-1}$ ) of the citric acid coated ferrophase dispersed in $\mathrm{KBr}$ (Fig. 3a) revealed bands from the iron citrate spectrum: (i) the large and intense band from $3450 \mathrm{~cm}^{-1}$ confirms the presence of water traces while the absorption at $3200-3400 \mathrm{~cm}^{-1}$ suggests the non-dissociated $\mathrm{OH}$ groups of the citric acid; (ii) the intense band at $1600 \mathrm{~cm}^{-1}$ may be assigned to the symmetric stretching of $\mathrm{OH}$ from $\mathrm{COOH}$ group while (iii) the neighbor band at $1400 \mathrm{~cm}^{-1}$ to the asymmetric stretchings of $\mathrm{CO}$ from $\mathrm{COOH}$ group can be assigned. The IR analysis of ferrophase coated with hydroxide of tetramethylammonium (Fig. 3b) revealed: (i) the $\mathrm{OH}$ group symmetrical and asymmetrical stretchings that generate the large band at about $3470 \mathrm{~cm}^{-1}$; (ii) the $\mathrm{CH}_{3}$ group from TMA molecule symmetrical and asymmetrical stretchings that gave the intense and bifurcated band at $2900 \mathrm{~cm}^{-1}$; (iii) the ferrophase complex vibrations that led to the $1300-1700 \mathrm{~cm}^{-1}$ bands and "skeleton" vibrations appeared within $400-900 \mathrm{~cm}^{-1}$. All the above mentioned features are in accordance with literature data [5].
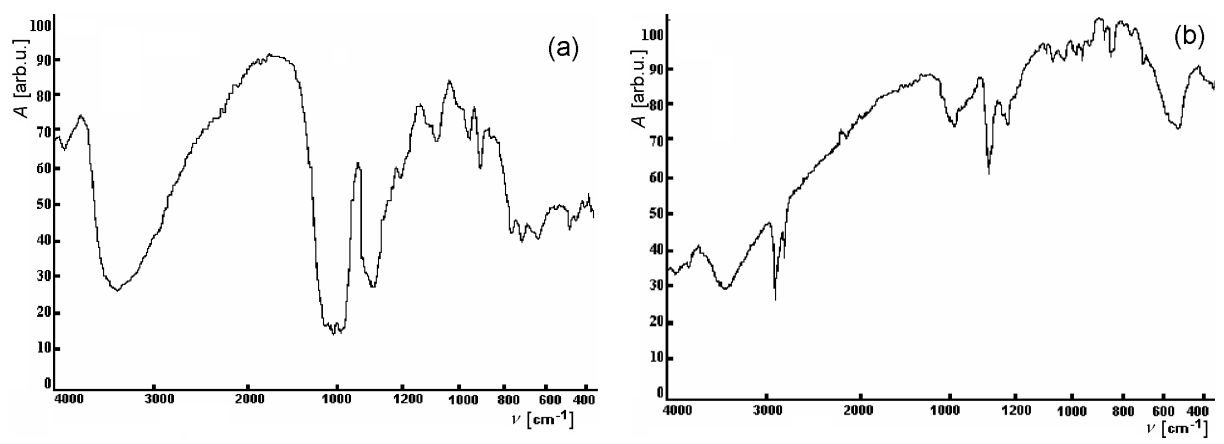

Fig. 3. (a) The IR spectrum of citric acid coated ferrophase; (b) the IR spectrum of ferrophase coated with tetramethylammonium hydroxide ( $A$ is the absorbance, while $\nu$ is the wave number).

The rheological features of the two products are presented by means of the data from Table. The characterization carried out on the two water ferrofluids revealed rather good ferrophase coating and consequently ferrofluid stability. In the next stages of our research the control of the magnetic properties is planned in order to get stable soft magnetic carriers. 
TABLE

Results of gravimetric, stalagmometric, and viscosity measurements.

\begin{tabular}{l|c|c|c}
\hline \hline & $\begin{array}{c}\text { Density } \\
{\left[\mathrm{kg} \mathrm{m}^{-3}\right]}\end{array}$ & $\begin{array}{c}\text { Surface tension } \\
{[\mathrm{N} / \mathrm{m}]}\end{array}$ & $\begin{array}{c}\text { Viscosity } \\
{\left[\mathrm{kg} \mathrm{m}^{-1} \mathrm{~s}^{-1}\right]}\end{array}$ \\
\hline CA-ferrofluid & 1126.87 & 0.05891 & $2.7083 \times 10^{-3}$ \\
TMA-ferrofluid & 1005.13 & 0.12965 & $2.8843 \times 10^{-3}$
\end{tabular}

\section{References}

[1] C. Rocchioli-Dectchoff, R. Frank, V. Cabuil, R.J. Massart, J. Chem. Res. 3, 67 (1967).

[2] C. Cotae, Z. Kelemen, N. Olaru, M. Cotae, Patent Ro, 93111, 1986.

[3] D. Creanga, Gh. Calugaru, J. Magn. Magn. Mater. 289, 81 (2005).

[4] R.E. Rosenweig, Ferrohydrodynamics, Cambridge University Press, New York 1985.

[5] D.S. Todorovsky, D.G. Dumanova, R.V. Todorovska, M.M. Getsova, Croatica Chem. Acta 75, 155 (2002). 